\title{
Conductive Organic Polymer Based Inorganic-Organic Hybrid Composite: Synthesis and Characterization
}

\author{
Anita Devi ${ }^{1, *}$, Honey Sharma ${ }^{2}$, Mukesh Kumar ${ }^{3}$ \\ ${ }^{1}$ Research Scholar, Department of Physical Science, Sant Baba Bhag Singh University, Jalandhar, \\ Punjab, India \\ ${ }^{2}$ Assistant Professor, Department of Physical Science, Sant Baba Bhag Singh University, Jalandhar, \\ Punjab, India \\ ${ }^{3}$ Assistant Professor, Department of Physics, Government Degree College, Billawar, Jammu and \\ Kashmir, India
}

\begin{abstract}
Inorganic organic (IO) hybrids where inserted conducting organic polymers (COP) imprint their polymeric nature onto the hybrid material are one of the most promising candidates for solving many technological problems of modern technocratic society. The Inorganic-Organic hybrid material of para toluidine (organic) and mercuric chloride (inorganic) was synthesized by using "instant-heating and slow-cooling" technique. Some light orange coloured plate-like crystals were obtained. XRD patterns of the grown crystal were analysed which shows that crystal has been grown into monoclinic crystal system in which $a \neq b \neq c$ [10.061(4) $\AA \neq 4.317(2) \AA \neq 12.987(5) \AA]$ and $\alpha=\gamma=90^{\circ} \neq \beta \quad\left[\alpha=\gamma=90^{\circ}, \quad \beta=104.322(7)^{\circ}\right]$ and belongs to space group $P 2{ }_{1}$. Analysis of secondary interactions shows that such material has potential application in opto-electronics.
\end{abstract}

Keywords: Conducting organic polymers, inorganic-organic hybrids, secondary interactions, slow cooling, optoelectronic devices, non-covalent weak interactions, hydrogen bond

*Author for Correspondence E-mail: anirajput035@gmail.com

\section{INTRODUCTION}

There is always a great need for better materials with predetermined multifunctional response. In this context, recent researches on inorganic-organic hybrid materials have shown that these materials have tremendous potential for solving various technological issues [1-5]. This is mainly due to the existence of innumerable possible combinations of organic and inorganic components with the purpose of tailoring resultant hybrid material's properties. Among all these possibilities, combination of conducting organic polymer with various inorganic components on the molecular scale has shown huge potential in various applications including energy storage, light sensitizer, solar cell and other optoelectronic applications [6-8]. In such hybrids, two species are linked through various intermolecular interactions including weak electrostatic interactions, hydrogen bonding, non-covalent bonding, covalent bonding, ionic bonding etc. These interactions play predominant role in defining the structure and the properties of the resultant hybrid material [9]. There are mainly two categories of such hybrid materials [10]. First is OrganicInorganic (OI) hybrid materials where the organic phase acts as host to an inorganic guest. In an organic-inorganic (OI) hybrid, conducting organic polymer (COP) acts as the host or major component and provides structural support to inorganic molecules to form hybrid structure. However inorganic component contributes in terms of its chemical activity. In OI hybrids, organic and inorganic components are linked together through covalent bonds. Second is Inorganic-Organic (IO) materials, with inorganic hosts and organic guests. In IO hybrids, inorganic component generally fulfils the task of structure formation, whereas inserted conducting organic polymer (COP) is for imprinting their polymeric nature into the 
resultant hybrid formed. Inspite of the fact that organic components have relatively large molecular weight, their role as intercalated guest molecule within Vander Waal gaps of layered or channel inorganic phases is highly recognized for structural stability of the resultant hybrid structure. Here we report the synthesis of para toluidine based hybrid material while taking mercuric chloride as inorganic component. Intermolecular secondary interaction analysis of the synthesized hybrid shows its potential application in optoelectronics [11-13].

\section{EXPERIMENTAL WORK}

$0.097 \mathrm{~g}$, i.e., $0.357 \mathrm{mmol}$ of mercuric chloride and $0.102 \mathrm{~g}$, i.e., $0.333 \mathrm{mmol}$ of para toluidine has been weighed out in a vial. $10 \mathrm{ml}(30 \%)$ of hydrochloric acid was added in the vial. Precipitates were formed and the sample was submersed in an ultrasonic bath but failed to form properly dissolved solution at room temperature. Vial was then heated in oil bath. The temperature of the oil bath was set to $90^{\circ} \mathrm{C}$ through programmable temperature controller provided with the oil bath. The temperature equals $90^{\circ} \mathrm{C}$ was kept constant for $4 \mathrm{~h}$. After then all precipitates were dissolved. A polytop lid sealing was made on the sample and finally vial height in the oil bath was adjusted. It was made in such a way that only filled part of the vial is immersed in the oil bath. After about $48 \mathrm{~h}$, slow cooling in the oil bath leads to the formation of crystals in the vial mostly on the upper half of the vial. Crystals were thin plate shaped and light orange in colour. Successfully prepared crystals were then extracted using filter paper. Philip PW 1315 powder diffractometer and CRYSFIRE programs has been used for obtaining X-Ray diffraction pattern and indexing of the pattern respectively [14-16].

\section{RESULTS AND DISCUSSION}

Crystal data of synthesized material showed that the grown crystal belongs to monoclinic crystal system in which $\mathrm{a} \neq \mathrm{b} \neq \mathrm{c}[10.061(4) \AA$ $\neq 4.317(2) \AA \neq 12.987(5) \AA]$ and $\alpha=\gamma=90^{\circ} \neq$ $\beta\left[\alpha=\gamma=90^{\circ}, \beta=104.322(7)^{\circ}\right]$ and belongs to space group $\mathrm{P} 2{ }_{1}$ as shown in Table 1. Sharp peaks of the powder diffraction pattern, as shown in Figure 1, confirms the crystallanity of the grown hybrid material. For inorganic part of the hybrid structure, mercury atoms and chlorine atoms are interconnected such that $\mathrm{Hg} 1-\mathrm{Cl} 1$ bond length is $2.354(2) \AA, \mathrm{Hg} 1$ $\mathrm{Cl} 2$ bond length is 2.352(1) $\AA$ and $\mathrm{Hg} 1-\mathrm{Cl} 3$ bond length is 2.925(7) $\AA$. The bond length with $\mathrm{Cl} 3$ is larger than rest of the two by the distance of $0.57 \AA$ which is quite significant distance. Also from the bond angle values for

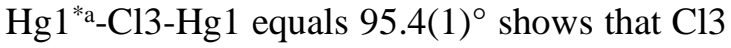
is acting as bifurcated acceptor so that inorganic part of the crystal structure forms a unique zig-zag pattern. $\mathrm{Cl} 1-\mathrm{Hg}-\mathrm{Cl} 2$ bond angle value is $173.2(1)$. Thus $\mathrm{Cl} 1$ and $\mathrm{Cl} 2$ are joined quite linearly through respective mercury atom. However all the angles with $\mathrm{Cl} 3$ are close to $90^{\circ}$ as shown in Figure 2.

Table 1: Crystal Data of Synthesized Material.

\begin{tabular}{|l|l|}
\hline \multicolumn{1}{|c|}{ Components of the Hybrid } & Paratoluidine (Organic), Mercuric Chloride (Inorganic) \\
\hline Empirical formula & $\mathrm{C}_{7} \mathrm{H}_{10} \mathrm{NHgCl}_{3}$ \\
\hline Formula weight & 415.10 \\
\hline Wavelength & $0.71073 \AA$ \\
\hline Crystal system & Monoclinic with unit cell parameters as \\
\hline $\mathrm{a}=10.061(4) \AA$ & $\alpha=90^{\circ}$ \\
\hline $\mathrm{b}=4.317(2) \AA$ & $\beta=104.322(7)^{\circ}$ \\
\hline $\mathrm{c}=12.987(5) \AA$ & $\gamma=90^{\circ}$ \\
\hline Space Group & $\mathrm{P} 21$ \\
\hline Absorption coefficient & $14.761 \mathrm{~mm}^{-1}$ \\
\hline Crystal size & $30 \mathrm{x} 28 \times 27 \mathrm{~mm}$ \\
\hline$\theta$ range for data collection & 1.62 to $26^{\circ}$ \\
\hline Miller Indices Limited to & $-12 \leq \mathrm{h} \leq 12,-5 \leq \mathrm{k} \leq 5,0 \leq \mathrm{l} \leq 16$ \\
\hline Reflections collected/unique & $2164 / 2164[\mathrm{R}(\mathrm{int})=0.0001]$ \\
\hline Completeness to Theta $=25.00$ & $99.9 \%$ \\
\hline Refinement Method & Full-matrix least-squares on $\mathrm{F}^{2}$ \\
\hline
\end{tabular}




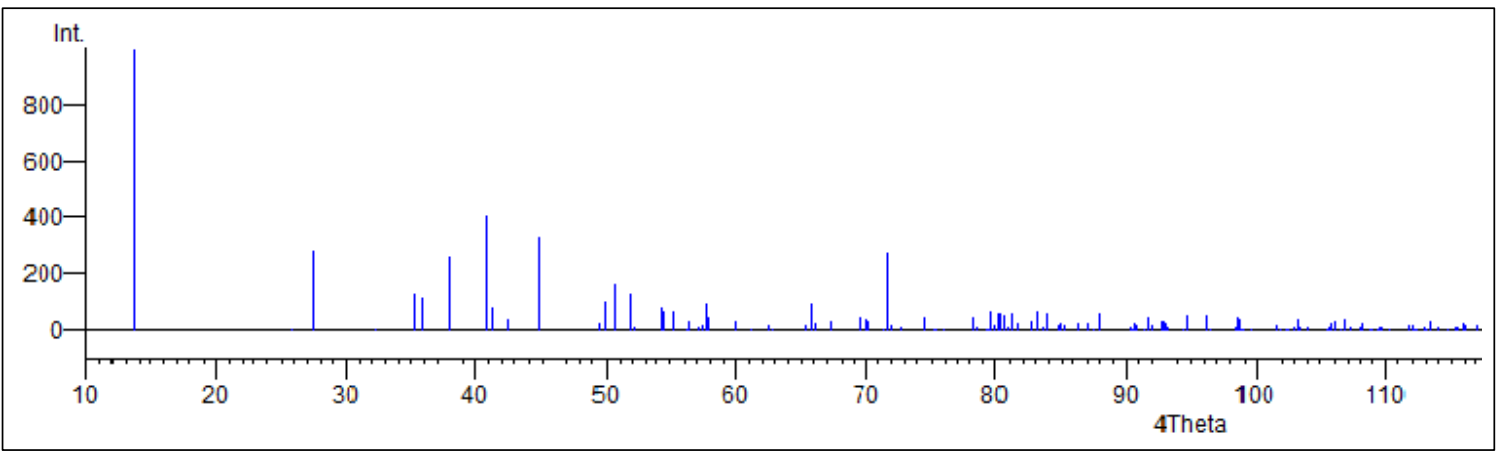

Fig. 1: Powder Diffraction Pattern of Synthesized Hybrid Material.

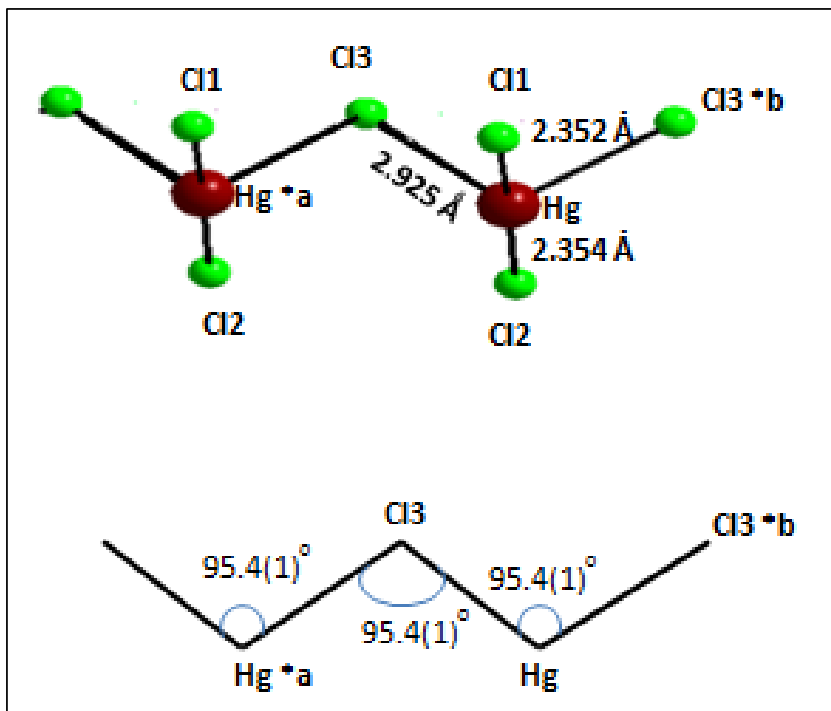

Fig. 2: Bond Angles and Bond Lengths Showing a Symmetric Zigzag Pattern Followed by Inorganic Moiety of the Hybrid Crystal.

The bond angles and bond lengths of the phenyl ring are quite comparable with the literaturevalues. The torsion angle for (C1-C6) of phenylring is of value $0.003(6)^{\circ}$ which is quite suffcient for its planar confirmation.

Furthermore, organic and inorganic moieties of the synthesized hybrid material have been observed to be linked together through $\mathrm{N}^{+}$$\mathrm{H}^{\cdots \cdots . .} \mathrm{Cl}^{-}$hydrogen bond as shown in Figure 3(a) through pink coloured dashed lines. Moreover these moieties are linked together in a unique tetramer pattern i.e. two inorganic moieties are linked to two organic

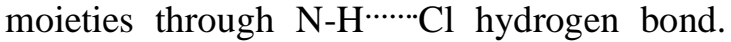
Single nitrogen atom of the organic moiety is acting as hydrogen bond donor for the formation of three hydrogen bonds. Also chlorine atoms i.e. $\mathrm{Cl} 3(\mathrm{x}, \mathrm{y}, \mathrm{z})$ and $\mathrm{Cl} 3(\mathrm{x}, \mathrm{y}-$ $1, \mathrm{z})$ are bifurcated acceptors for two hydrogen atoms and the bifurcated acceptor angle is $\mathrm{H}_{\mathrm{A}^{-}}$
$\mathrm{Cl} 3-\mathrm{H}_{\mathrm{B}}$ is $23.76(2)^{\circ}$. When seen along acplane, tetramer pattern have been observed to be extended along the diagonal of ac-plane. One tetramer pattern is linked to another through $\mathrm{Hg}-\mathrm{Cl}$ metal halide weak interaction as depicted in Figure 2(b). Mercury atom $\mathrm{Hg} 1(-1+\mathrm{x}, 3+\mathrm{y}, \mathrm{z})$ is connected to chlorine atom Cl1 $(1-\mathrm{x}, 5 / 2 \mathrm{y}, \mathrm{z})$ via bond distance of 3.135(6) $\AA$. Since the sum of their Vander Waal radii is $3.30 \AA$, therefore metal-halide weak interaction is confirmed. These secondary metal-halide interactions are of the kind dipole-dipole interaction, i.e., $\mathrm{Hg}^{+\cdots \cdots . . .} \mathrm{Cl}^{-}$ and the strength as calculated from the bond distance vs. bond valence model is just $5-10 \%$ of the $\mathrm{Hg}-\mathrm{Cl}$ bond. This $\mathrm{Hg}{ }^{\cdots \cdots} \mathrm{Cl}$ weak interaction resulted into the formation of a symmetric dimmer pattern of inorganic moiety. When minimum distance existing between the two mercury atoms is measured, it comes out to be 4.112(1) $\AA$. 


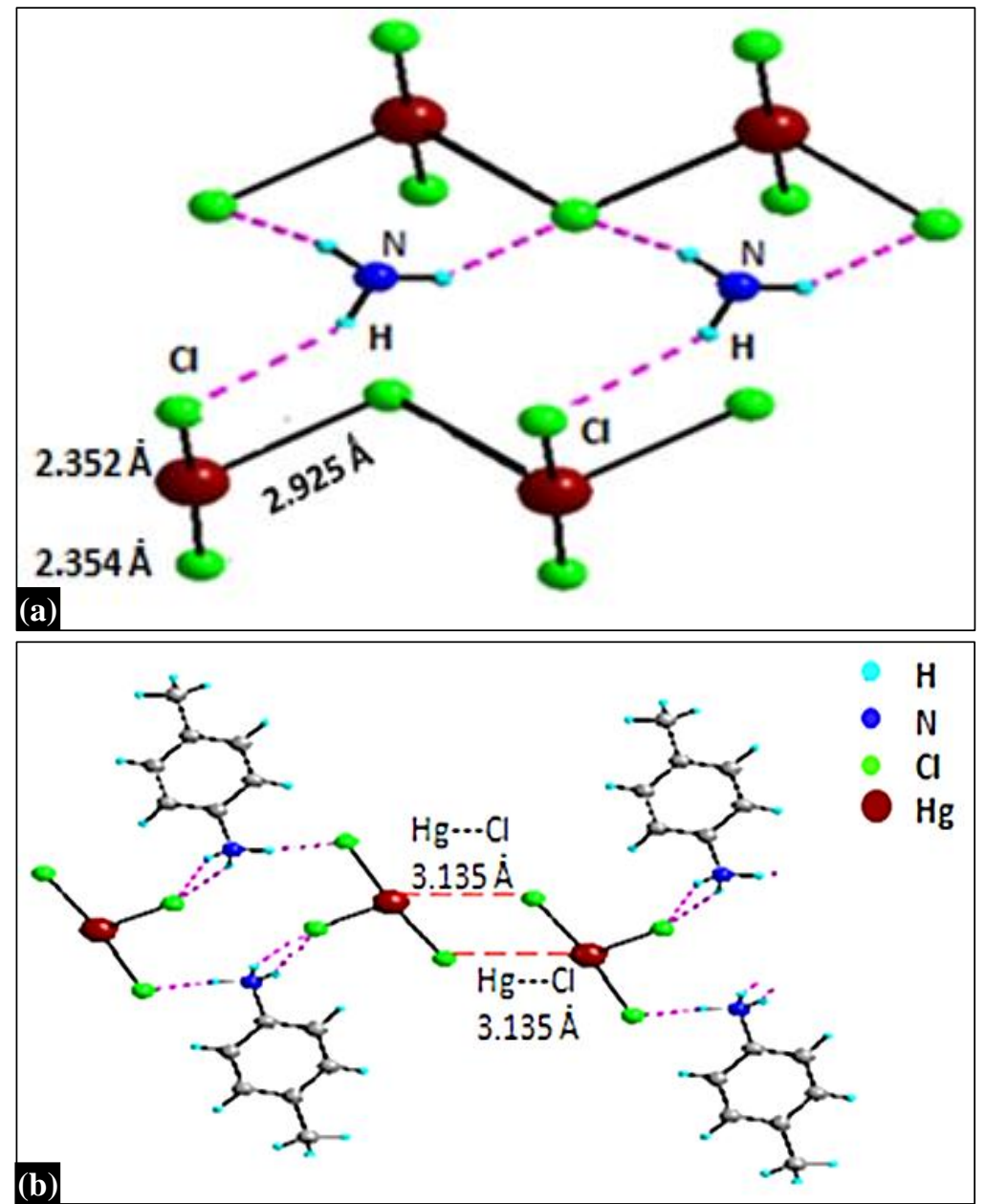

Fig. 3: Perspective View of (a) Hydrogen Bonding (Pink Dashed Line), (b) Metal Halide Secondary Weak Interaction (Red Dashed Line) through which Organic and Inorganic Components are Linked Together to Form a Single Composite.

However the sum of their Vander Waal radii is approximately $3.50 \AA$. Thus, non-existence of mercurophilic interaction, i.e., $\mathrm{Hg}-\mathrm{Hg}$ interaction in this compound is confirmed. Similarly there is no halogen-halogen interaction as the minimum $\mathrm{Cl}-\mathrm{Cl}$ distance is 3.764(7) A. Also the distance from the centroid of aromatic ring to the nearest hydrogen atom comes out to be 3.695(2) $\AA$ which is quite large for $\mathrm{C}-\mathrm{H} \cdots \cdots \cdot \pi$ interaction to exist [17-19].

\section{CONCLUSION}

The inorganic (mercuric chloride) organic (para-toluidine) hybrid material was synthesized by using slow cooling technique. The light orange coloured crystals were obtained. The powder diffraction peak profiles for the hybrid material shows that $b$-axis has smaller value as compared to other two axes. The torsion angle of phenyl ring comes out to be $0.003(6)^{\circ}$ which gives its planar confirmation for synthesized inorganic-organic hybrid material. Organic and inorganic moieties of the synthesized hybrid material are

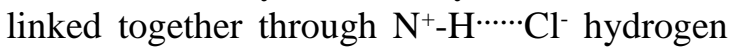
bond. Two inorganic moieties are linked to two organic moieties through $\mathrm{N}-\mathrm{H}^{\cdots \cdots \cdot .} \mathrm{Cl}$ hydrogen bond to form unique tetramer pattern. Layers of inorganic and organic moieties are linked through $\mathrm{Hg}-\mathrm{Cl}$ metal halide weak interaction. There is no metallophilic interaction as the minimum $\mathrm{Hg}$ $\mathrm{Hg}$ distance comes out to be 4.112(1) $\AA$ which is quite large for any metallophilic interaction to exist. The minimum halide-halide distance is 3.764(7) $\AA$ which indicates that there is hardly any halogen-halogen interaction. 


\section{REFERENCES}

1. Mir SH, Nagaharo LA, Thundat T, et al. Functional Materials: An Integrated Platform for Applied Technologies. $J$ Electrochem Soc.2018; 165(8):B3137-56p.

2. Nanko M. Definition and Categories of Hybrid Materials. Adv. in. tech. of mat. and mat. Proc. J. 2019; 11(1):1-8p.

3. $\mathrm{Gu} \mathrm{H}$, Liu $\mathrm{C}$, Zhu J, et al. Introducing Advanced Composites and Hybrid Materials. Adv Compos Hybrid Mater. 2018; 1(1):1-5p.

4. Kalia S, Haldorai Y. Advances in Polymer Science. (Vol. 267). Switzerland: Springer Nature; 2019.

5. Pagliaro M, Nedelec JM. Hybrid Materials. Nanoscale.2014; 6(12):6219p.

6. Aronwalsh. Principles of Chemical Bonding and Band Gap Engineering in Organic Inorganic Halide Perovskite. $J$ Phys Chem C.2015; 119(11):5755-60p.

7. Reiss $\mathrm{P}$, Aldakov D. Organic Inorganic Hybrid Materials for Optoelectronics. Synth Met.2016; 222 part A: 1-2p.

8. Zhang $\mathrm{F}, \mathrm{Lu} \mathrm{H}$, Tong J, et al. Advances in Two Dimensional Organic-Inorganic Hybrid Perovskite. Energy Environ Sci. Advance Article, 2020. https://doi.org/ 10.1039/C9EE03757H.

9. Desiraju GR. Crystal Engineering: A Brief Overview. $J$ Chem Sci. 2010; 122(5):667-75p.

10. Romero PG. Hybrid Organic Inorganic Materials-in Search of Synergic Activity. Adv Materials. 2001; 13(3):163-72p.

11. Mellouhi FE, Bentria T, Marzouk A, et al. Hydrogen Bonding: A Mechanism for
Tuning Electronic and Optical Properties of Hybrid Organic-Inorganic Frameworks. npjComput Mater.2016;2:16035p.

12. Kickelbick G. Hybrid Materials- Past, Present and Future. Hybrid Mater. 2014; 1(1):39-51p.

13. Liang D, Roelkens $\mathrm{G}$, Baets $\mathrm{R}$, et al. Hybrid Integrated Platform for Silicon Photonics. Materials.2010; 3(3):1782-02p.

14. Bruker. SMART, SAINT-Plus and SADABS. Madison, Wisconsin, USA: Bruker AXS Inc.; 1998.

15. Sheldrick GM. SHELXS97, Program for Crystal Structure Solution. Germany: University of G"ottingen; 1997.

16. Sheldrick GM. SHELXL97, Program for Crystal Structure Refinement. Germany: University of G*ottingen; 1997.

17. Rahim Z, Barman BN. The Van der Waals Criterion for Hydrogen Bonding. ActaCryst.1978; A(34):761-4p.

18. Batsanov AS. Weak Interactions in Crystals: Old Concepts, New Developments. Acta Cryst. 2018; $\mathrm{E}(74): 570-4 \mathrm{p}$.

19. Batsanov SS. Van der Waals Radii of Elements. InorgMater.2001; 37(9):871-5p.

\section{Cite this Article}

Anita Devi, Honey Sharma, Mukesh Kumar. Conductive Organic Polymer Based Inorganic-Organic Hybrid Composite: Synthesis and Characterization. Journal of Polymer \& Composites. 2020; 8(1): 1-5p. 